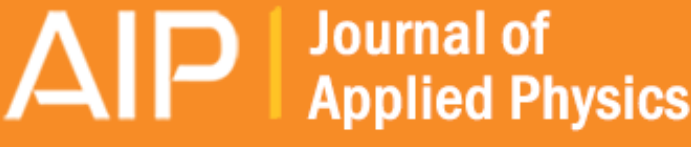

\section{A theory of electroded thin thermopiezoelectric plates subject to large driving voltages}

\author{
J. S. Yang and R. C. Batra
}

Citation: Journal of Applied Physics 76, 5411 (1994); doi: 10.1063/1.358489

View online: http://dx.doi.org/10.1063/1.358489

View Table of Contents: http://scitation.aip.org/content/aip/journal/jap/76/9?ver=pdfcov

Published by the AIP Publishing

\section{Articles you may be interested in}

Voltage dependent photocurrent of thin film hematite electrodes

Appl. Phys. Lett. 99, 063508 (2011); 10.1063/1.3622130

Electroelastic equations for electroded thin plates subject to large driving voltages

J. Appl. Phys. 74, 3389 (1993); 10.1063/1.354565

Theory of vibrations of coated, thermopiezoelectric laminae

J. Math. Phys. 19, 109 (1978); 10.1063/1.523527

Theory of vibrations of coated, thermopiezoelectric laminae

J. Acoust. Soc. Am. 60, S47 (1976); 10.1121/1.2003365

Analysis of the operation of electret transducers subject to large electrode displacements J. Acoust. Soc. Am. 55, 345 (1974); 10.1121/1.1914509

MIT LINCOLN LABORATORY CAREERS

Discover the satisfaction of innovation and service to the nation
- Space Control

- Air \& Missile Defense

- Communications Systems \& Cyber Security

- Intelligence, Surveillance and Reconnaissance Systems

- Advanced
Electronics
- Tactical Systems
- Homeland
Protection
- Air Traffic Control

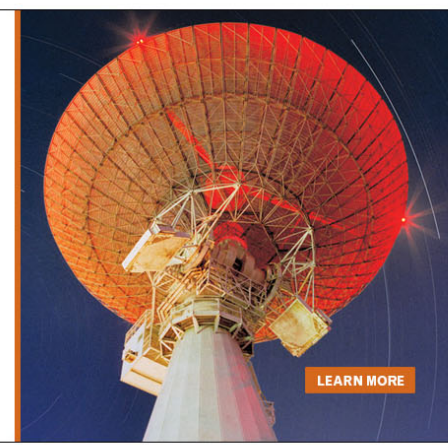




\title{
A theory of electroded thin thermoplezoelectric plates subject to large driving voltages
}

\author{
J. S. Yang \\ Department of Mechanical Engineering, Rensselaer Polytechnic Institute, Troy, New York 12180 \\ R. C. Batra \\ Clifton Garvin Professor, Department of Engineering Science and Mechanics, Virginia Polytechnic Institute \\ and State University, Blacksburg, Virginia 24061-0219
}

(Received 23 May 1994; accepted for publication 14 July 1994)

\begin{abstract}
We first reduce existing equations for nonlinear thermoelectroelasticity to the case of large electric fields, small strains, and infinitesimal temperature variations. These equations are then specialized to the case of thin plates with completely electroded major surfaces, and it is shown that in this case the charge equation of electrostatics is satisfied trivially to the lowest order. An example is given to illustrate the effect of heat conduction on the response of a thin electroded plate subjected to time harmonic voltages on the two major surfaces.
\end{abstract}

\section{INTRODUCTION}

Piezoelectric materials in intelligent structures are often subjected to strong driving voltages. Based on his general formulation for nonlinear thermoelectroelasticity, ${ }^{1}$ Tiersten $^{2}$ has recently derived a set of equations quadratic in electric fields that are suitable for studying deformations of piezoelectric materials subjected to strong driving voltages. $\mathrm{He}$ has also specialized these equations to fully electroded very thin plates. $^{2}$ These equations are also used to derive a set of two-dimensional equations for piezoelectric plates subjected to strong driving voltages, ${ }^{3}$ and to analyze a composite elastic plate with piezoelectric actuators. ${ }^{4}$

In Ref. 2, only the piezoelectric effect is considered. In a recent review article, ${ }^{5}$ Rao and Sunar have pointed out that the composite intelligent structures have different response characteristics at different temperatures ${ }^{6}$ and the temperature variation in piezoelectric materials can noticeably affect the overall performance of the control system. ${ }^{7}$ Hence, thermal effects are important in the precision distributed sensing and control of intelligent structures. According to Rao and Sunar ${ }^{5}$ applications of thermopiezoelectricity theory to practical engineering problems in general, and to vibration control of flexible structures in particular, are very few in the literature. ${ }^{8-10}$ Also, the development of the thermopiezoelectric sensors/actuators is important for advanced intelligent structures.

In this article, equations derived in Ref. 2 are generalized to include thermal effects. The nonlinear constitutive relations for a thermopiezoelectric material linear in small strains and temperature variations, but quadratic in the relatively strong electric field, are obtained by retaining proper terms in the expansion of an energy density function. The complete set of the nonlinear thermopiezoelectric equations quadratic in electric fields are then specialized to the case of a thin thermopiezoelectric plate with fully electroded major surfaces.

\section{EQUATIONS FOR NONLINEAR THERMOPIEZOELECTRICITY}

Let the coordinates of a material particle with respect to a rectangular Cartesian coordinate system be $X_{M}$ in the ref- erence configuration and $y_{i}$ in the current configuration. The motion of the material point is described by $y_{i}=y_{i}\left(X_{L}, t\right)$, where $t$ is the time. Throughout this article, a repeated index implies summation over the range of the index, and a comma followed by $M$ (or $i$ ) implies partial differentiation with respect to $X_{M}$ (or $y_{i}$ ). A dot above a quantity denotes its material time derivative.

The equations governing the finite deformations of a nonlinear thermopiezoelectric body $\operatorname{arc}^{1,2}$

$$
\begin{aligned}
& \rho^{0}=\rho J, \quad K_{L j, L}=\delta_{j M} \rho^{0} \ddot{u}_{M}, \quad \mathscr{Q}_{L, L}=0, \\
& -Q_{L, L}=\rho^{0} T \dot{\eta}, \quad-Q_{L} T_{, L} \geqslant 0, \\
& K_{L j}=H_{L j}+M_{L j}, \quad M_{L j}=J X_{L, i} \epsilon_{0}\left(E_{i} E_{j}-\frac{1}{2} E_{k} E_{k} \delta_{i j}\right), \\
& \mathscr{D}_{L}=\epsilon_{0} \mathscr{E}_{L}+\mathscr{P}_{L}, \quad \mathscr{E}_{L}=J X_{L, i} E_{i}, \\
& H_{L j}=\rho^{0} y_{j, M} \frac{\partial \chi}{\partial E_{L M}}, \quad \mathscr{P}_{L}=-\rho^{0} \frac{\partial \chi}{\partial W_{L}}, \quad \eta=-\frac{\partial \chi}{\partial T}, \\
& \chi=\chi\left(E_{K L} ; W_{L} ; T\right), \quad Q_{K}=Q_{K}\left(T_{, M} ; E_{L M} ; W_{L} ; T\right), \\
& J=\operatorname{det}\left(y_{i, L}\right), \quad E_{K L}=\frac{1}{2}\left(y_{i, L} y_{i, M}-\delta_{L M}\right), \\
& E_{i}=-\phi_{, i}, \quad W_{L}=y_{i, L} E_{i}=-\phi_{, L},
\end{aligned}
$$

where $\rho^{0}$ and $\rho$ are the mass densities in the reference and present configurations, $K_{L j}$ is the total Piola-Kirchhoff stress tensor, $\delta_{j M}$ is a translation operator which serves to translate a vector from the present to the reference configuration and vice versa and is required for notational consistency and clarity because of the use of upper- and lower-case indices, respectively, to refer to the reference and present positions of a material point, $u_{M}$ is the mechanical displacement vector, $\mathscr{D}_{L}$ the reference electric displacement, $Q_{L}$ the reference heat flux vector, $T$ the absolute temperature, $\eta$ the entropy per unit mass, $\epsilon_{0}$ the electric permittivity of free space in mks units, $E_{i}$ the electric field, $\delta_{i j}$ the Kronecker delta, $E_{K L}$ the material strain tensor, and $\phi$ the electric potential. Equations in the first line of (1) represent the conservation of mass, the balance of linear momentum, and the electric charge equa- 
tion. Equations in the second line, i.e., the heat equation and the entropy inequality are consequences of the first and second laws of thermodynamics. Equations in the fifth and sixth lines of (1) are the constitutive relations which are determined by the specification of two functions $\chi$ and $Q_{K}$ and the form of $Q_{K}$ is restricted by the entropy inequality.

The boundary conditions at the material surface in the reference configuration with unit exterior normal $N_{L}$ involve the specification of either $u_{M}$ or $N_{L} K_{L j}, \phi$ or $N_{L} \mathscr{D}_{L}$, and $T$ or $N_{L} Q_{L}$.

The purpose of this work is to derive a set of equations for the case of small strain infinitesimal temperature variations, and strong electric fields. The only nonlinear terms to be kept are those quadratic in the electric field, as in Ref. 2. For this purpose, the scalar function $\chi$ may be expanded with respect to the reference state of zero strain, zero electric field, and uniform reference temperature $T_{0}$ :

$$
\begin{aligned}
\rho^{0} \chi\left(E_{K L} ; W_{L} ; T\right)= & \frac{1}{2} c_{A B C D} E_{A B} E_{C D}-\frac{1}{2} \chi_{A B} W_{A} W_{B} \\
& -\frac{1}{2} \rho^{0} C\left(T-T_{0}\right)^{2}-e_{A B C} W_{A} E_{B C} \\
& -\alpha_{A B} E_{A B}\left(T-T_{0}\right)-p_{A} W_{A}\left(T-T_{0}\right) \\
& -\frac{1}{2} b_{A B C D} W_{A} W_{B} E_{C D}-\frac{1}{2} \gamma_{A B} W_{A} W_{B}(T \\
& \left.-T_{0}\right)-\frac{1}{6} \chi_{A B C} W_{A} W_{B} W_{C}
\end{aligned}
$$

where the material constants $c_{A B C D}, e_{A B C}, \chi_{A B}, b_{A B C D}$, and $\chi_{A B C}$ are called the elastic, piezoelectric, second-order electric permeability, electrostrictive, and third-order electric permeability, respectively. $\alpha_{A B}$ and $p_{A}$ are the thermoelastic and pyroelectric constants, respectively. $C$ is the specific heat, and $\gamma_{A B}$ are nonlinear pyroelectric constants. In Eq. (2) we have assumed that the body is stress free in the reference configuration and have accordingly excluded the term linear in $E_{A B}$ on the right-hand side. The material constants satisfy symmetry relations, e.g., $c_{A B C D}=c_{C D A B}=c_{B A C D}=c_{A B D C}$.

Equation (2) results in the following constitutive relations:

$$
\begin{aligned}
H_{L j}=y_{j, M} \frac{\partial \rho^{0} \chi}{\partial E_{L M}}= & y_{j, M}\left(c_{L M C D} E_{C D}-e_{A L M} W_{A}-\alpha_{L M} \theta\right. \\
& \left.-\frac{1}{2} b_{A B L M} W_{A} W_{B}\right), \\
\mathscr{P}_{L}=-\frac{\partial \rho^{0} \chi}{\partial W_{L}}= & e_{L B C} E_{B C}+\chi_{L B} W_{B}-p_{L} \theta+\frac{1}{2} \chi_{L B C} W_{B} W_{C} \\
& +b_{L B C D} W_{B} E_{C D}+\gamma_{L B} W_{B} \theta \\
\rho^{0} \eta=-\frac{\partial \rho^{0} \chi}{\partial T}= & \alpha_{A B} E_{A B}+p_{A} W_{A}+\rho^{0} C \theta \\
& +\frac{1}{2} \gamma_{A B} W_{A} W_{B},
\end{aligned}
$$

where we have set the temperature variation $T-T_{0}=\theta$. We note that in Eq. (3) $)_{2}$ there are two terms involving the product of $W_{B}$ and $E_{C D}$, and the product of $W_{B}$ and $\theta$. Since a theory quadratic in electric field variables is desired, we drop these two terms and obtain

$$
\begin{aligned}
H_{L j}= & y_{j, M}\left(c_{L M C D} E_{C D}-e_{A L M} W_{A}-\alpha_{L M} \theta\right. \\
& \left.-\frac{1}{2} b_{A B L M} W_{A} W_{B}\right),
\end{aligned}
$$

$$
\begin{aligned}
& \mathscr{P}_{L}=e_{L B C} E_{B C}+\chi_{L B} W_{B}+p_{L} \theta+\frac{1}{2} \chi_{L B C} W_{B} W_{C}, \\
& \rho^{0} \eta=\alpha_{A B} E_{A B}+p_{A} W_{A}+\rho^{0} C \theta+\frac{1}{2} \gamma_{A B} W_{A} W_{B} .
\end{aligned}
$$

We note that although Eq. (3) has some unneeded terms for the present purpose, it has the advantage of being derived from an energy density function $\chi$. The removal of the unneeded terms results in the simpler form Eq. (4), which serves the present purpose well but does not admit a scalar generating function. The existence of an energy density or generating function is important in variational and related numerical methods.

The simplest form of the function $Q_{K}$ which satisfies the entropy inequality is ${ }^{1}$

$$
Q_{K}\left(T_{, M} ; E_{L M} ; W_{L} ; T\right)=-\kappa_{K N}\left(E_{L M} ; W_{L} ; T\right) T_{, N},
$$

where $\kappa_{K N}$ is positive definite, however $\kappa_{K N}$ may still depend on $E_{K L}, W_{L}$, and $T$. Since we are keeping only those nonlinear terms that are quadratic in the electric field, henceforth we assume that $\kappa_{K N}$ does not depend on $E_{K L}, W_{L}$, and $T$. Hence

$$
Q_{K}\left(T_{, M} ; E_{L M} ; W_{D} ; T\right)=-\kappa_{K N}\left(0 ; 0 ; T_{0}\right) \theta_{, N},
$$

where the constant matrix $\kappa_{K N}$ is positive definite.

Equations (4) and (6) are the constitutive relations we need to consider, which are quadratic in the electric field variables and satisfy the entropy inequality.

\section{SMALL STRAINS AND TEMPERATURE VARIATIONS, AND QUADRATIC ELECTRIC FIELDS}

We now reduce the general thermopiezoelectric equations given in the previous section to the special case of infinitesimal strains and temperature variations, and quadratic electric fields. In making the reduction, powers of $u_{M}$, $\theta$, and their gradients higher than the first are dropped in all expressions, and even the linear terms themselves are dropped in comparison with any finite quantity, such as the Kronecker delta and 1 , in the usual manner. In this way we obtain $^{2}$

$$
\begin{aligned}
& y_{i, K} \rightarrow \delta_{i K}, \quad X_{L, i} \rightarrow \delta_{L i}, \quad J \rightarrow 1, \\
& E_{L M} \rightarrow S_{L M}=\frac{1}{2}\left(u_{L, M}+u_{M, L}\right),
\end{aligned}
$$

where the symbol $\rightarrow$ implies replaced by. Thus the constitutive relations in Eq. (4) simplify to

$$
\begin{aligned}
H_{L j}= & \delta_{j M}\left(\sigma_{L M C D} S_{C D}-e_{A L M} W_{A}-\alpha_{L M} \theta\right. \\
& \left.-\frac{1}{2} b_{A B L M} W_{A} W_{B}\right), \\
\mathscr{P}_{L}= & e_{L B C} S_{B C}+\chi_{L B} W_{B}+p_{L} \theta+\frac{1}{2} \chi_{L B C} W_{B} W_{C}, \\
\rho^{0} \eta= & \alpha_{A B} S_{A B}+p_{A} W_{A}+\rho^{0} C \theta+\frac{1}{2} \gamma_{A B} W_{A} W_{B}, \\
Q_{K}= & -\kappa_{K N} \theta_{, N} .
\end{aligned}
$$

With Eq. (7) and the relevant equations in Eq. (1) we have

$$
\begin{aligned}
& M_{L j}=\delta_{j M} \epsilon_{0}\left(W_{L} W_{M}-\frac{1}{2} W_{K} W_{K} \delta_{L M}\right), \\
& \mathscr{E}_{L}=W_{L} .
\end{aligned}
$$




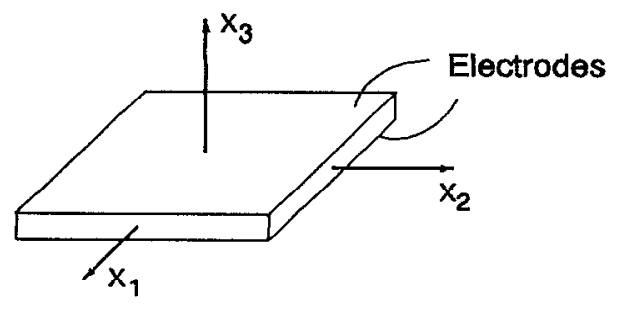

FIG. 1. A fully electroded thin thermopiezoelectric plate.

The substitution of Eqs. (8) and (9) into the relevant equations in Eq. (1) yields

$$
\begin{aligned}
& K_{L j}=\delta_{j M} T_{L M}, \\
& T_{L M}=c_{L M A B} S_{A B}-e_{A L M} W_{A}-\alpha_{L M} \theta-\frac{1}{2} \hat{b}_{A B L M} W_{A} W_{B}, \\
& \hat{b}_{A B L M}=b_{A B L M}-2 \epsilon_{0}\left(\delta_{L A} \delta_{M B}-\frac{1}{2} \delta_{A B} \delta_{L M}\right), \\
& \mathscr{V}_{L}=e_{L B C} S_{B C}+\epsilon_{L A} W_{A}+p_{L} \theta+\frac{1}{2} \chi_{A B L} W_{A} W_{B}, \\
& \epsilon_{L A}=\epsilon_{0} \delta_{L A}+\chi_{L A} .
\end{aligned}
$$

Substitution of Eq. (10) $)_{1}$ into the stress equations of motion in Eq. (1) gives

$$
T_{L M, L}=p^{0} \ddot{u}_{M} \text {. }
$$

Within our approximation, we can also write

$$
-Q_{L, L}=\rho^{0}\left(T_{0}+\theta\right) \dot{\eta} \approx \rho^{0} T_{0} \dot{\eta} \text {. }
$$

In summary, we have obtained

$$
\begin{aligned}
& T_{L M, L}=\rho^{0} \ddot{u}_{M}, \quad \mathscr{D}_{L, L}=0, \quad-Q_{L, L}=T_{0} \rho^{0} \dot{\eta}, \\
& T_{L M}=c_{L M A B} S_{A B}-e_{A L M} W_{A}-\alpha_{L M} \theta-\frac{1}{2} \hat{b}_{A B L M} W_{A} W_{B}, \\
& \mathscr{Q}_{L}=e_{L B C} S_{B C}+\epsilon_{L A} W_{A}+p_{L} \theta+\frac{1}{2} \chi_{A B L} W_{A} W_{B}, \\
& \rho^{0} \eta=\alpha_{A B} S_{A B}+p_{A} W_{A}+\rho^{0} C \theta+\frac{1}{2} \gamma_{A B} W_{A} W_{B}, \\
& Q_{L}=-\kappa_{L M} \theta_{, M}, \\
& S_{L M}=\frac{1}{2}\left(u_{L, M}+u_{M, L}\right), \quad W_{L}=-\phi_{, L},
\end{aligned}
$$

which is the desired theory for small strains and temperature variations, and strong electric fields. We note that Eq. (13) reduces to the nonlinear theory of piezoelectricity quadratic in electric fields given in Rcf. 2 , and the linear theory of thermopiezoelectricity given in Ref. 11 . The substitution of Eq. (13) ${ }_{2-6}$ into Eq. (13) $)_{1}$ yields five equations for the five unknowns $u_{M}, \phi$, and $\theta$.

\section{EXTENSIONAL EQUATIONS FOR THIN PLATES WITH ELECTRODED SURFACES}

We now consider a thin plate with fully electroded major surfaces such as that shown in Fig. 1, which also depicts the coordinate system used to study deformations of the plate. The major surfaces of the plate are assumed to be traction free and thermally insulated. Since we consider infinitesimal strains, we dispense with the no longer necessary convention that capital indices refer to the reference coordinates and lower-case indices refer to the present coordinates of material points, and use lower-case indices exclusively to refer quantities to the reference coordinates of material points. In addition, for convenience and because it is conventional to do so, we introduce the usual compressed notation for tensor indices for stress and strain in the constitutive relations, in which $p, q, r$, and $s$ take values $1,2,3,4,5,6$ as $i j$ or $k l$ take values $11,22,33,23$ or 32,31 or 13,12 or 21 . In the compressed notation, constitutive relations $(13)_{2-5}$ become

$$
\begin{aligned}
& T_{p}=c_{p q} S_{q}-e_{k p} E_{k}-\alpha_{p} \theta-\frac{1}{2} \hat{b}_{k j p} E_{k} E_{j}, \\
& D_{l}=e_{l q} S_{q}+\epsilon_{l k} E_{k}+p_{l} \theta+\frac{1}{2} \chi_{k j} E_{k} E_{j}, \\
& \rho^{0} \eta=\alpha_{q} S_{q}+p_{k} E_{k}+\rho^{0} C \theta+\frac{1}{2} \gamma_{k j} E_{k} E_{j}, \\
& Q_{l}=-\kappa_{l k} \theta_{, k},
\end{aligned}
$$

where we have taken the liberty of writing $D_{l}$ for $\mathscr{D}_{L}$ and $E_{l}$ for $W_{L}$ since the distinction between them is lost when the assumption of infinitesimal strains is employed.

It is common to assume that the electroded top and bottom surfaces are thermally insulated. Therefore, the heat flux $Q_{3}$ on the electroded surfaces vanishes and since the plate is very thin, we may assume that $Q_{3}=0$ throughout the plate. From the third equation of Eq. (14) 4 we obtain

$$
Q_{3}=-\kappa_{31} \theta_{, 1}-\kappa_{32} \theta_{, 2}-\kappa_{33} \theta_{, 3}=0,
$$

with which the first two equations of Eq. (14) ${ }_{4}$ can be written as

$$
\begin{aligned}
& Q_{a}=-\kappa_{a b}^{p} \theta_{, b}, \\
& \kappa_{11}^{p}=\kappa_{11}-\kappa_{13} \kappa_{13} / \kappa_{33}, \\
& \kappa_{12}^{p}=\kappa_{12}-\kappa_{13} \kappa_{23} / \kappa_{33}, \quad \kappa_{22}^{p}=\kappa_{22}-\kappa_{23} \kappa_{23} / \kappa_{33},
\end{aligned}
$$

where $a, b, c$, and $d$ take values 1 and 2 only. The heat equation assumes the form

$$
-Q_{a, a}=T_{0} \rho^{0} \dot{\eta} .
$$

Similarly, since the tractions $T_{3 m}$ on the electroded major surfaces vanish and the plate is very thin, we assume that the state of stress corresponds to that of plane stress and take $T_{3 m}=0$ throughout the plate, and $T_{a b}=T_{a b}\left(X_{a}, t\right)$. Since $T_{3 m}=0, \ddot{u}_{3}$ must be negligible, which is always the case for the extensional deformations of thin plates. Thus for extensional deformations of the thin plate,

$$
T_{a b, a}=\rho^{0} \ddot{u}_{b} \text {. }
$$

With $T_{3 m}=0$, it is advantageous to obtain strain-stress relations. ${ }^{2}$ To this end we operate on Eq. $(14)_{1}$ with $c_{r p}^{-1}=s_{r p}$ to obtain

$$
S_{q}=s_{q p} T_{p}+d_{k q} E_{k}+\alpha_{q}^{T} \theta+\frac{1}{2} \beta_{j k q} E_{j} E_{k},
$$

where

$$
d_{k q}=e_{k p} s_{p q}, \quad \alpha_{q}^{T}=\alpha_{p} s_{p q}, \quad \beta_{j k q}=\hat{b}_{j k p} s_{p q}
$$

and we note that $d_{k q}$ and $\alpha_{q}^{T}$ are well-known sets of material constants ${ }^{12}$ which are useful for plane stress type calculations, and $\beta_{j k q}$ is as in Ref. 2. The substitution of Eq. (20) into Eq. (14) $)_{2,3}$ yields

$$
\begin{aligned}
& D_{l}=d_{l p} T_{p}+\epsilon_{l k}^{T} E_{k}+p_{l}^{T} \theta+\frac{1}{2} \chi_{j k l}^{T} E_{j} E_{k}, \\
& \rho^{0} \eta=\alpha_{p}^{T} T_{p}+p_{k}^{T} E_{k}+\rho^{0} C^{T} \theta+\frac{1}{2} \gamma_{j k}^{T} E_{j} E_{k},
\end{aligned}
$$




$$
\begin{aligned}
& \epsilon_{l k}^{T}=\epsilon_{l k}+e_{l q} d_{k q}, \quad p_{l}^{T}=p_{l}+e_{l q} \alpha_{q}^{T}, \\
& \chi_{j k l}^{T}=\chi_{j k l}+e_{l q} \beta_{j k q}, \\
& \rho^{0} C^{T}=\rho^{0} C+\alpha_{q} \alpha_{q}^{T}, \quad \gamma_{j k}^{T}=\gamma_{j k}+\alpha_{q} \beta_{j k q}
\end{aligned}
$$

and we note that $\epsilon_{l k}^{T}, p_{l}^{T}$, and $C^{T}$ are well-known material constants, ${ }^{12} \chi_{j k l}^{T}$ is as in Ref. 2 , and $\gamma_{j k}^{T}$ is new.

Since $T_{3 m}=0$, the constitutive relations Eqs. (19) and (21) may be reduced to

$$
\begin{aligned}
& S_{q}=s_{q v} T_{v}+d_{k q} E_{k}+\alpha_{q}^{T} \theta+\frac{1}{2} \beta_{j k q} E_{j} E_{k}, \\
& D_{l}=d_{l v} T_{v}+\epsilon_{l k}^{T} E_{k}+p_{l}^{T} \theta+\frac{1}{2} \chi_{j k l}^{T} E_{j} E_{k}, \\
& \rho^{0} \eta=\alpha_{v}^{T} T_{v}+p_{k}^{T} E_{k}+\rho^{0} C^{T} \theta+\frac{1}{2} \gamma_{j k}^{T} E_{j} E_{k},
\end{aligned}
$$

where $u, v$ assume values $1,2,6$, but not $3,4,5$.

Next, since the electric fields vanish in the conducting electrodes and the plate is thin, we may take $E_{a}=0$ throughout the plate. ${ }^{2}$ Then only $E_{3}$ will appear in Eq. (23) and $\beta_{j k q}$, $\chi_{j k l}^{T}, \gamma_{j k}^{T}$ will assume $\beta_{33 q}, \chi_{33 l}^{T}, \gamma_{33}^{T}$ which can be further compressed as

$$
\beta_{33 q} \rightarrow \beta_{3 q}, \quad \chi_{33 l}^{T} \rightarrow \chi_{3 l}^{T}, \quad \gamma_{33}^{T} \rightarrow \gamma_{3}^{T}
$$

with which Eqs. (23) become

$$
\begin{aligned}
& S_{q}=s_{q v} T_{v}+d_{3 q} E_{3}+\alpha_{q}^{T} \theta+\frac{1}{2} \beta_{3 q} E_{3}^{2}, \\
& D_{l}=d_{l v} T_{v}+\epsilon_{l 3}^{T} E_{3}+p_{l}^{T} \theta+\frac{1}{2} \chi_{3 l}^{T} E_{3}^{2}, \\
& \rho^{0} \eta=\alpha_{v}^{T} T_{v}+p_{3}^{T} E_{3}+\rho^{0} C^{T} \theta+\frac{1}{2} \gamma_{3}^{T} E_{3}^{2} .
\end{aligned}
$$

The first, second, and sixth of the unabbreviated Eq. $(25)_{1}$ are

$$
S_{u}=s_{u v} T_{v}+d_{3 u} E_{3}+\alpha_{u}^{T} \theta+\frac{1}{2} \beta_{3 u} E_{3}^{2},
$$

which can be solved to give

$$
T_{u}=c_{u v}^{p} S_{v}-e_{3 u}^{p} E_{3}-\alpha_{u}^{p} \theta-\frac{1}{2} \hat{b}_{3 u}^{p} E_{3}^{2},
$$

where $c_{u v}^{p}$ is the inverse of the $3 \times 3$ matrix $s_{u v}$, and

$$
e_{l u}^{p}=d_{l v} c_{v u}^{p}, \quad \alpha_{u}^{p}=c_{u v}^{p} \alpha_{v}^{T}, \quad \hat{b}_{3 u}^{p}=c_{u v}^{p} \beta_{3 u} .
$$

The substitution of Eq. (27) into Eq. $(25)_{2,3}$ yields

$$
\begin{aligned}
& D_{l}=e_{l u}^{p} S_{u}+\epsilon_{l 3}^{p} E_{3}+p_{l}^{p} \theta+\frac{1}{2} \chi_{3 l}^{p} E_{3}^{2}, \\
& \rho^{0} \eta=\alpha_{u}^{p} S_{u}+p_{3}^{p} E_{3}+\rho^{0} C^{p} \theta+\frac{1}{2} \gamma_{3}^{p} E_{3}^{2},
\end{aligned}
$$

where

$$
\begin{aligned}
& \epsilon_{l 3}^{p}=e_{l 3}^{T}-d_{l v} e_{3 v}^{p}, \quad p_{l}^{p}=p_{l}^{T}-d_{l v} \alpha_{v}^{p}, \\
& \chi_{3 l}^{p}=\chi_{3 l}^{T}-d_{l v} \hat{b}_{3 v}^{p}, \\
& \rho^{0} C^{p}=\rho^{0} C^{T}-\alpha_{v}^{p} \alpha_{v}^{T}, \quad \gamma_{3}^{p}=\gamma_{3}^{T}-\alpha_{v}^{T} \hat{b}_{3 v}^{p} .
\end{aligned}
$$

Finally, in the present notation the electric charge equation takes the form

$$
D_{l, l}=0 \text {. }
$$

Since for the thin plate shown in Fig. $1, D_{l}$ vary slowly with respect to $X_{a}$, we first write Eq. (31) in the form

$$
D_{3,3}+D_{a, a}=0 \text {, }
$$

and then note that $D_{a, a}$ is of smaller order than $D_{3,3}$. Hence, to the lowest order we have ${ }^{2}$

$$
D_{3,3}=0 \text {, }
$$

which yields

$$
D_{3}=D_{3}\left(X_{a}, t\right) \text {. }
$$

Furthermore, since for a state of plane-stress to the lowest order $S_{v}$ is independent of $X_{3}$, and $\theta$ in Eq. (29) 1 is independent of $X_{3}$ to the lowest order, ${ }^{13}$ Eqs. (33) and (29) imply that

$$
E_{3,3}=0 \text {, }
$$

which yields

$$
E_{3}=-V / 2 b,
$$

where $V$ is the applied voltage across the plate and $2 b$ the plate thickness.

In summary, we have

$$
\begin{aligned}
& T_{a b, a}=\rho^{0} \ddot{u}_{b}, \quad-Q_{a, a}=T_{0} \rho^{0} \dot{\eta}, \\
& T_{u}=c_{u v}^{p} S_{v}-e_{3 u}^{p} E_{3}-\alpha_{u}^{p} \theta-\frac{1}{2} \hat{b}_{3 u}^{p} E_{3}^{2}, \\
& \rho^{0} \eta=\alpha_{u}^{p} S_{u}+p_{3}^{p} E_{3}+\rho^{0} C^{p} \theta+\frac{1}{2} \gamma_{3}^{p} E_{3}^{2}, \\
& Q_{a}=-\kappa_{a b}^{p} \theta_{, b}, \\
& S_{a b}=\frac{1}{2}\left(u_{a, b}+u_{b, a}\right), \quad E_{3}=-V / 2 b, \\
& D_{3}=e_{3 u}^{p} S_{u}+\epsilon_{33}^{p} E_{3}+p_{3}^{p} \theta+\frac{1}{2} \chi_{33}^{p} E_{3}^{2},
\end{aligned}
$$

which can be written as three equations for the three unknowns $u_{a}\left(X_{a}, t\right)$ and $\theta\left(X_{a}, t\right)$. These equations generalize those given in Ref. 2, and can be used to model piezoelectric actuators with known $V(t)$ in smart structures when heat effects need to be considered. We have also included the expression of $D_{3}$ in Eq. (37) which is needed when the thin plate is used as a sensor in which case $V$ is unknown and $D_{3}$ is used to determine the surface charge density on the electrodes, then $V$ can be computed through the capacitance of the thin plate. The indices in Eq. (37) take the following values:

$$
a, b, c, d=1,2 ; \quad u, v=1,2,6 .
$$

\section{EQUATIONS FOR A THIN CERAMIC PLATE}

For a ceramic plate poled in the $X_{3}$ direction, we have $\mathrm{e}^{12}$

$$
\left(s_{p q}\right)=\left(\begin{array}{cccccc}
s_{11} & s_{12} & s_{13} & 0 & 0 & 0 \\
s_{12} & s_{11} & s_{13} & 0 & 0 & 0 \\
s_{13} & s_{13} & s_{33} & 0 & 0 & 0 \\
0 & 0 & 0 & s_{44} & 0 & 0 \\
0 & 0 & 0 & 0 & s_{44} & 0 \\
0 & 0 & 0 & 0 & 0 & s_{66}
\end{array}\right),
$$

where $s_{66}=2\left(s_{11}-s_{12}\right)$, and

$$
\begin{aligned}
& \left(d_{k q}\right)=\left(\begin{array}{cccccc}
0 & 0 & 0 & 0 & d_{15} & 0 \\
0 & 0 & 0 & d_{15} & 0 & 0 \\
d_{31} & d_{31} & d_{33} & 0 & 0 & 0
\end{array}\right), \\
& \left(p_{k}^{T}\right)=\left(0,0, p_{3}^{T}\right),
\end{aligned}
$$




$$
\left(\epsilon_{k l}^{T}\right)=\left(\begin{array}{ccc}
\epsilon_{11}^{T} & 0 & 0 \\
0 & \epsilon_{11}^{T} & 0 \\
0 & 0 & \epsilon_{33}^{T}
\end{array}\right),
$$

with $\kappa_{k l}, \gamma_{k l}^{T}$, and $\alpha_{k l}^{T}$ having the same structure as $\epsilon_{k l}^{T}$. Hence

$$
\left(\alpha_{q}^{T}\right)=\left(\alpha_{1}^{T}, \alpha_{1}^{T}, \alpha_{3}^{T}, 0,0,0\right),
$$

and we note that since $\kappa_{13}=\kappa_{23}=0$ we have $\kappa_{a b}^{p}=\kappa_{a b}$.

From Eq. $(25)_{1}$ and the matrices given in the appendix of Ref. 14 we conclude that the only component of $\beta_{j k q}$ needed is $\beta_{331}=\beta_{332}$ which is compressed as $\beta_{31}$ with its value determined in Ref. 2 from experimental data as $0.7949 \mathrm{pm}^{2} / \mathrm{V}^{2}$ for PZT G-1195.

With Eqs. (39)-(43) we have

$$
\begin{aligned}
& S_{1}=s_{11} T_{1}+s_{12} T_{2}+d_{31} E_{3}+\alpha_{1}^{T} \theta+\frac{1}{2} \beta_{31} E_{3}^{2}, \\
& S_{2}=s_{12} T_{1}+s_{11} T_{2}+d_{31} E_{3}+\alpha_{1}^{T} \theta+\frac{1}{2} \beta_{31} E_{3}^{2}, \\
& S_{6}=s_{66} T_{6},
\end{aligned}
$$

which can be inverted to give

$$
\begin{aligned}
& T_{1}=c_{11}^{p} S_{1}+c_{12}^{p} S_{2}+e_{31}^{p} E_{3}+\alpha_{1}^{p} \theta+\frac{1}{2} \hat{b}_{31}^{p} E_{3}^{2}, \\
& T_{2}=c_{12}^{p} S_{1}+c_{11}^{p} S_{2}+e_{31}^{p} E_{3}+\alpha_{1}^{p} \theta+\frac{1}{2} \hat{b}_{31}^{p} E_{3}^{2}, \\
& T_{6}=c_{66} S_{6},
\end{aligned}
$$

where

$$
\begin{aligned}
& c_{11}^{p}=s_{11} / \Delta, \quad c_{12}^{p}=-s_{12} / \Delta, \quad \Delta=s_{11}^{2}-s_{12}^{2}, \\
& e_{31}^{p}=d_{31} /\left(s_{11}+s_{12}\right), \quad \alpha_{1}^{p}=\left(c_{11}^{p}+c_{12}^{p}\right) \alpha_{1}^{T}, \\
& \hat{b}_{31}^{p}=\beta_{31} /\left(s_{11}+s_{12}\right) .
\end{aligned}
$$

Substitution of Eq. (45) into Eq. (30) gives

$$
\begin{aligned}
& p_{3}^{p}=p_{3}^{T}-2 d_{31} \alpha_{1}^{p}, \quad \rho^{0} C^{p}=\rho^{0} C^{T}-2 \alpha_{1}^{p} \alpha_{1}^{T}, \\
& \gamma_{3}^{p}=\gamma_{3}^{T}-2 \alpha_{1}^{T} \hat{b}_{31}^{p}, \\
& \epsilon_{33}^{p}=\epsilon_{33}^{T}-2 d_{31} e_{31}^{p}, \quad \chi_{33}^{p}=\chi_{33}^{T}-2 d_{31} \hat{b}_{31}^{p},
\end{aligned}
$$

which yields

$$
\begin{aligned}
& \rho^{0} \eta=\alpha_{1}^{p}\left(S_{1}+S_{2}\right)+p_{3}^{p} E_{3}+\rho^{0} C^{p} \theta+\frac{1}{2} \gamma_{3}^{p} E_{3}^{2}, \\
& D_{3}=e_{31}^{p}\left(S_{1}+S_{2}\right)+e_{33}^{p} E_{3}+p_{3}^{p} \theta+\frac{1}{2} \chi_{33}^{p} E_{3}^{2} .
\end{aligned}
$$

Hence, for a ceramic plate poled in the $X_{3}$ direction, Eq. (37) becomes

$$
\begin{aligned}
& T_{a b, a}=\rho^{0} \ddot{u}_{b}, \quad-Q_{a, a}=T_{0} \rho^{0} \dot{\eta}, \\
& T_{1}=c_{11}^{p} S_{1}+c_{12}^{p} S_{2}+e_{31}^{p} E_{3}+\alpha_{1}^{p} \theta+\frac{1}{2} \hat{b}_{31}^{p} E_{3}^{2}, \\
& T_{2}=c_{12}^{p} S_{1}+c_{11}^{p} S_{2}+e_{31}^{p} E_{3}+\alpha_{1}^{p} \theta+\frac{1}{2} \hat{b}_{31}^{p} E_{3}^{2}, \\
& T_{6}=c_{66} S_{6}, \\
& \rho^{0} \eta=\alpha_{1}^{p}\left(S_{1}+S_{2}\right)+p_{3}^{p} E_{3}+\rho^{0} C^{p} \theta+\frac{1}{2} \gamma_{3}^{p} E_{3}^{2}, \\
& Q_{1}=-\kappa_{11} \theta_{, 1}, \quad Q_{2}=-\kappa_{11} \theta_{, 2}, \\
& S_{a b}=\frac{1}{2}\left(u_{a, b}+u_{b, a}\right), \quad E_{3}=-V / 2 b, \\
& D_{3}=e_{31}^{p}\left(S_{1}+S_{2}\right)+e_{33}^{p} E_{3}+p_{3}^{p} \theta+\frac{1}{2} \chi_{33}^{p} E_{3}^{2} .
\end{aligned}
$$

\section{EQUATIONS FOR A THIN PVDF PLATE} have ${ }^{12}$

For a thin plate of PVDF which is of class $m m 2$, we

$$
\left(s_{p q}\right)=\left(\begin{array}{cccccc}
s_{11} & s_{12} & s_{13} & 0 & 0 & 0 \\
s_{12} & s_{22} & s_{13} & 0 & 0 & 0 \\
s_{13} & s_{23} & s_{33} & 0 & 0 & 0 \\
0 & 0 & 0 & s_{44} & 0 & 0 \\
0 & 0 & 0 & 0 & s_{55} & 0 \\
0 & 0 & 0 & 0 & 0 & s_{66}
\end{array}\right)
$$

and

$$
\begin{aligned}
& \left(d_{k q}\right)=\left(\begin{array}{cccccc}
0 & 0 & 0 & 0 & d_{15} & 0 \\
0 & 0 & 0 & d_{24} & 0 & 0 \\
d_{31} & d_{32} & d_{33} & 0 & 0 & 0
\end{array}\right), \\
& \left(p_{k}^{T}\right)=\left(0,0, p_{3}^{T}\right), \\
& \left(\epsilon_{k l}^{T}\right)=\left(\begin{array}{ccc}
\epsilon_{11}^{T} & 0 & 0 \\
0 & \epsilon_{22}^{T} & 0 \\
0 & 0 & \epsilon_{33}^{T}
\end{array}\right),
\end{aligned}
$$

with $\kappa_{k l}, \gamma_{k l}^{T}$ and $\alpha_{k l}^{T}$ having the same structure as $\epsilon_{k l}^{T}$. Hence

$$
\left(\alpha_{q}^{T}\right)=\left(\alpha_{1}^{T}, \alpha_{2}^{T}, \alpha_{3}^{T}, 0,0,0\right),
$$

and since $\kappa_{13}=\kappa_{23}=0$, we have $\kappa_{a b}^{p}=\kappa_{a b}$.

From Eq. $(25)_{1}$ and the matrices given in the appendix of Ref. 14 the only components of $\beta_{j k q}$ needed are $\beta_{331}$ and $\beta_{332}$ which are compressed as $\beta_{31}$ and $\beta_{32}$.

With Eqs. (50)- $-(54)$ we have

$$
\begin{aligned}
& S_{1}=s_{11} T_{1}+s_{12} T_{2}+d_{31} E_{3}+\alpha_{1}^{T} \theta+\frac{1}{2} \beta_{31} E_{3}^{2}, \\
& S_{2}=s_{12} T_{1}+s_{22} T_{2}+d_{32} E_{3}+\alpha_{2}^{T} \theta+\frac{1}{2} \beta_{32} E_{3}^{2}, \\
& S_{6}=s_{66} T_{6},
\end{aligned}
$$

which can be inverted to give

$$
\begin{aligned}
& T_{1}=c_{11}^{p} S_{1}+c_{12}^{p} S_{2}+e_{31}^{p} E_{3}+\alpha_{1}^{p} \theta+\frac{1}{2} \hat{b}_{31}^{p} E_{3}^{2}, \\
& T_{2}=c_{12}^{p} S_{1}+c_{22}^{p} S_{2}+e_{32}^{p} E_{3}+\alpha_{2}^{p} \theta+\frac{1}{2} \hat{b}_{32}^{p} E_{3}^{2}, \\
& T_{6}=c_{66} S_{6},
\end{aligned}
$$

where

$c_{11}^{p}=s_{22} / \Delta, \quad c_{22}^{p}=s_{11} / \Delta, \quad c_{12}^{p}=-s_{12} / \Delta$,

$\Delta=s_{11} s_{22}-s_{12}^{2}$,

$e_{31}^{p}=c_{11}^{p} d_{31}+c_{12}^{p} d_{32}, \quad e_{32}^{p}=c_{12}^{p} d_{31}+c_{22}^{p} d_{32}$,

$\alpha_{1}^{p}=c_{11}^{p} \alpha_{1}^{T}+c_{12}^{p} \alpha_{2}^{T}, \quad \alpha_{2}^{p}=c_{11}^{p} \alpha_{1}^{T}+c_{22}^{p} \alpha_{2}^{T}$,

$\hat{b}_{31}^{p}=c_{11}^{p} \beta_{31}+c_{12}^{p} \beta_{32}, \quad \hat{b}_{32}^{p}=c_{12}^{p} \beta_{31}+c_{22}^{p} \beta_{32}$.

Substitution of Eq. (56) into Eq. (30) gives

$p_{3}^{p}=p_{3}^{T}-d_{31} \alpha_{1}^{p}-d_{32} \alpha_{2}^{p}$,

$\rho^{0} C^{p}=\rho^{0} C^{T}-\alpha_{1}^{p} \alpha_{1}^{T}-\alpha_{2}^{p} \alpha_{2}^{T}$,

$\gamma_{3}^{p}=\gamma_{3}^{T}-\alpha_{1}^{T} \hat{b}_{31}^{p}-\alpha_{2}^{T} \hat{b}_{32}^{p}, \quad \epsilon_{33}^{p}=\epsilon_{33}^{T}-d_{31} e_{31}^{p}-d_{32} e_{32}^{p}$,

$\chi_{33}^{p}=\chi_{33}^{T}-d_{31} \hat{b}_{31}^{p}-d_{32} \hat{b}_{32}^{p}$,

which yield 


$$
\begin{aligned}
& \rho^{0} \eta=\alpha_{1}^{p} S_{1}+\alpha_{2}^{p} S_{2}+p_{3}^{p} E_{3}+\rho^{0} C^{p} \theta+\frac{1}{2} \gamma_{3}^{p} E_{3}^{2}, \\
& D_{3}=e_{31}^{p} S_{1}+e_{32}^{p} S_{2}+\epsilon_{33}^{p} E_{3}+p_{3}^{p} \theta+\frac{1}{2} \chi_{33}^{p} E_{3}^{2} .
\end{aligned}
$$

Hence, for a plate of PVDF, Eq. (37) becomes

$$
\begin{aligned}
& T_{a b, a}=\rho^{0} \ddot{u}_{b}, \quad-Q_{a, a}=T_{0} \rho^{0} \dot{\eta}, \\
& T_{1}=c_{11}^{p} S_{1}+c_{12}^{p} S_{2}+e_{31}^{p} E_{3}+\alpha_{1}^{p} \theta+\frac{1}{2} \hat{b}_{31}^{p} E_{3}^{2}, \\
& T_{2}=c_{12}^{p} S_{1}+c_{22}^{p} S_{2}+e_{32}^{p} E_{3}+\alpha_{2}^{p} \theta+\frac{1}{2} \hat{b}_{32}^{p} E_{3}^{2}, \\
& T_{6}=c_{66} S_{6}, \\
& \rho^{0} \eta=\alpha_{1}^{p} S_{1}+\alpha_{2}^{p} S_{2}+p_{3}^{p} E_{3}+\rho^{0} C^{p} \theta+\frac{1}{2} \gamma_{3}^{p} E_{3}^{2}, \\
& Q_{1}=-\kappa_{11} \theta_{, 1}, \quad Q_{2}=-\kappa_{22} \theta_{, 2}, \\
& S_{a b}=\frac{1}{2}\left(u_{a, b}+u_{b, a}\right), \quad E_{3}=-V / 2 b, \\
& D_{3}=e_{31}^{p} S_{1}+e_{32}^{p} S_{2}+e_{33}^{p} E_{3}+p_{3}^{p} \theta+\frac{1}{2} \chi_{33}^{p} E_{3}^{2} .
\end{aligned}
$$

\section{AN EXAMPLE}

As an example, we consider a ceramic plate poled in the $X_{3}$ direction. The plate is finite in the $X_{1}$ direction with $-l<X_{1}<l$, and infinite in the $X_{2}$ direction. Like an actuator, the plate is assumed to be in one-dimensional extensional motion with $u_{2}=0$ and $\partial / \partial X_{2}=0$. The ends $X_{1}=-l, l$ are traction free and are thermally insulated. The driving voltage $V$ is assumed to be time harmonic with $V=\bar{V} e^{i \omega t}$, where $\bar{V}$ and $\omega$ are known, real constants and only the real part of $V$ is of physical interest. Then the relevant equations out of Eq. (49) and with the appropriate boundary conditions are

$$
\begin{aligned}
& T_{1,1}=\rho^{0} \ddot{u}_{1}, \quad-Q_{1,1}=T_{0} \rho^{0} \dot{\eta}, \quad-l<X_{1}<l, \\
& T_{1}=c_{11}^{p} u_{1,1}+e_{31}^{p} E_{3}+\alpha_{1}^{p} \theta+\frac{1}{2} \hat{b}_{31}^{p} E_{3}^{2}, \quad-l<X_{1}<l, \\
& \rho^{0} \eta=\alpha_{1}^{p} u_{1,1}+p_{3}^{p} E_{3}+\rho^{0} C^{p} \theta+\frac{1}{2} \gamma_{3}^{p} E_{3}^{2}, \quad-l<X_{1}<l, \\
& Q_{1}=-\kappa_{11} \theta_{, 1}, \quad-l<X_{1}<l, \\
& c_{11}^{p} u_{1,1}+\alpha_{1}^{p} \theta=-e_{31}^{p} E_{3}-\frac{1}{2} \hat{b}_{31}^{p} E_{3}^{2} \text { at } X_{1}=-l, l, \\
& -\kappa_{11} \theta_{, 1}=0 \text { at } X_{1}=-l, l, \\
& E_{3}=-\bar{V} e^{i \omega t} / 2 b .
\end{aligned}
$$

Since in Eq. (61) the quadratic term $E_{3}^{2}$ is prescribed, the problem is linear. The steady sate solution to $\mathrm{Eq}$. (61) can be found with some algebra but no essential difficulties. The results are

$$
\begin{aligned}
u_{1}\left(X_{1}, t\right)= & \left(\frac{\alpha_{1}^{p} p_{3}^{p}}{\rho^{0} C^{p}}-e_{31}^{p}\right) \frac{\bar{V}}{2 b} \frac{\pi_{2} k_{2} \sin \left(k_{2} l\right) \sin \left(k_{1} X_{1}\right)-\pi_{1} k_{1} \sin \left(k_{1} l\right) \sin \left(k_{2} X_{1}\right)}{\pi_{1} k_{1}\left(c_{11}^{p} k_{2}+\alpha_{1}^{p} \pi_{2}\right) \sin \left(k_{1} l\right) \cos \left(k_{2} l\right)-\pi_{2} k_{2}\left(c_{11}^{p} k_{1}+\alpha_{1}^{p} \pi_{1}\right) \cos \left(k_{1} l\right) \sin \left(k_{2} l\right)} e^{i \omega t} \\
& +\left(\frac{b_{31}^{p}}{2}-\frac{\alpha_{1}^{p} \gamma_{3}^{p}}{2 \rho^{0} C^{p}}\right)\left(\frac{\bar{V}}{2 b}\right)^{2} \frac{\pi_{4} k_{4} \sin \left(k_{4} l\right) \sin \left(k_{3} X_{1}\right)-\pi_{3} k_{3} \sin \left(k_{3} l\right) \sin \left(k_{4} X_{1}\right)}{\pi_{3} k_{3}\left(c_{11}^{p} k_{4}+\alpha_{1}^{p} \pi_{4}\right) \sin \left(k_{3} l\right) \cos \left(k_{4} l\right)-\pi_{4} k_{4}\left(c_{11}^{p} k_{3}+\alpha_{1}^{p} \pi_{3}\right) \cos \left(k_{3} l\right) \sin \left(k_{4} l\right)} e^{i 2 \omega t}, \\
\theta\left(X_{1}, t\right)= & \frac{p_{3}^{p}}{\rho^{0} C^{p}} \frac{\bar{V}}{2 b} e^{i \omega t}+\left(\frac{\alpha_{1}^{p} p_{3}^{p}}{\rho^{0} C^{p}}-e_{31}^{p}\right) \frac{\bar{V}}{2 b} \\
& \times \frac{\pi_{1} \pi_{2} k_{2} \sin \left(k_{2} l\right) \cos \left(k_{1} X_{1}\right)-\pi_{1} \pi_{2} k_{1} \sin \left(k_{1} l\right) \cos \left(k_{2} X_{1}\right)}{\pi_{1} k_{1}\left(c_{11}^{p} k_{2}+\alpha_{1}^{p} \pi_{2}\right) \sin \left(k_{1} l\right) \cos \left(k_{2} l\right)-\pi_{2} k_{2}\left(c_{11}^{p} k_{1}+\alpha_{1}^{p} \pi_{1}\right) \cos \left(k_{1} l\right) \sin \left(k_{2} l\right)} e^{i \omega t} e^{i 2 \omega t}, \\
& -\frac{\gamma_{3}^{p}}{2 \rho^{0} C^{p}}\left(\frac{\bar{V}}{2 b}\right)^{2} e^{i 2 \omega t}+\left(\frac{b_{31}^{p}}{2}-\frac{\alpha_{1}^{p} \gamma_{3}^{p}}{2 \rho^{0} C^{p}}\right)\left(\frac{\bar{V}}{2 b}\right)^{2} \\
& \left.\times \frac{\pi_{3} \pi_{4} k_{4} \sin \left(k_{4} l\right) \cos \left(k_{3} X_{1}\right)-\pi_{3} \pi_{4} k_{3} \sin \left(k_{3} l\right) \cos \left(k_{4} X_{1}\right)}{\pi_{3} k_{3}\left(c_{11}^{p} k_{4}+\alpha_{1}^{p} \pi_{4}\right) \sin \left(k_{3} l\right) \cos \left(k_{4} l\right)-\pi_{4} k_{4}\left(c_{11}^{p} k_{3}+\alpha_{1}^{p} \pi_{3}\right) \cos \left(k_{3} l\right) \sin \left(k_{4} l\right)} e^{2}{ }^{2}\right)
\end{aligned}
$$

where $k_{1}^{2}$ and $k_{2}^{2}$ are the two roots of the following equation for $k^{2}$ :

$$
\left(\rho^{0} \omega^{2}-c_{11}^{p} k^{2}\right)\left(\kappa_{11} k^{2}+T_{0} \rho^{0} C^{p} i \omega\right)+i \omega T_{0}\left(\alpha_{1}^{p} k\right)^{2}=0
$$

and

$$
\pi_{1}=\frac{\rho^{0} \omega^{2}-c_{11}^{p} k_{1}^{2}}{\alpha_{1}^{p} k_{1}}, \quad \pi_{2}=\frac{\rho^{0} \omega^{2}-c_{11}^{p} k_{2}^{2}}{\alpha_{1}^{p} k_{2}} .
$$

Similarly, $k_{3}^{2}$ and $k_{4}^{2}$ are the two roots of the following equation for $k^{2}$ :

$\left(\rho^{0} 4 \omega^{2}-c_{11}^{p} k^{2}\right)\left(\kappa_{11} k^{2}+T_{0} \rho^{0} C^{p} i 2 \omega\right)+i 2 \omega T_{0}\left(\alpha_{1}^{p} k\right)^{2}=0$ and

$$
\pi_{3}=\frac{\rho^{0} 4 \omega^{2}-c_{11}^{p} k_{3}^{2}}{\alpha_{1}^{p} k_{3}}, \quad \pi_{4}=\frac{\rho^{0} 4 \omega^{2}-c_{11}^{p} k_{4}^{2}}{\alpha_{1}^{p} k_{4}}
$$

In Eqs. (63)-(66), $k_{1}-k_{4}$ and $\pi_{1}-\pi_{4}$ are all complex. Hence $u_{1}$ and $\theta$ in Eq. (62) have both complex amplitudes and complex wave numbers $k$. The real part of Eq. (62) represents our physical process. It can be seen that $V$ excites a motion symmetric about the center $X_{1}=0$. The solution consists of two parts with frequencies $e^{i \omega t}$ and $e^{i 2 \omega t}$, respectively.

The quadratic term $E_{3}^{2}$ contributes to the part with $e^{i 2 \omega t}$ 
factor which does not exist in the results of the linear theory of thermopiezoelectricity.

The part with the $e^{i \omega t}$ factor is the result of the linear theory of thermopiezoelectricity. Comparing with the results of the linear theory of piezoelectricity,

$$
\begin{aligned}
& u_{1}\left(X_{1}, t\right)= \frac{e_{31}^{p}(\bar{V} / 2 b)}{c_{11}^{p} \sqrt{\rho^{0} / c_{11}^{p}} \omega \cos \left(\sqrt{\rho^{0} / c_{11}^{p}} \omega l\right)} \\
& \times \sin \left(\sqrt{\frac{\rho^{0}}{c_{11}^{p}}} \omega X_{1}\right) e^{i \omega t}, \\
& \theta\left(X_{1}, t\right)=0,
\end{aligned}
$$

we see that the thermal effect alters not only the amplitude and wavenumber but also the phase of the motion, as is the case when damping enters the system. Here the damping is due to the irreversible heat conduction process. Because of the complex wavenumber, the $X_{1}$ dependence of the thermopiezoelectric solution will have both exponential and sinusoidal behavior. This is different from Eq. (67).

\section{CONCLUSIONS}

We have derived a theory of thermopiezoelectricity for the case of large electric fields but small strains and small temperature variations. These equations are specialized to the case of thin plates with completely electroded major surfaces. In this case the charge equation of electrostatics is satisfied trivially to the lowest order. The thin plate equations can be used to model piezoelectric actuators or sensors when thermal effects need to be considered. The specific forms of thin plate equations are given for piezoelectric materials PZT and PVDF. An analytical example shows that when quadratic electric field and thermal effect are considered, the qualitative behavior of the solution differs noticeably from the solution of linear piezoelectricity.

\section{ACKNOWLEDGMENTS}

This work was supported by the U.S. Army Research Office Grant No. DAAH 04-93-G-0284 to the University of Missouri-Rolla, and a matching grant from the Missouri Research and Training Center.

${ }^{1}$ H. F. Tiersten, Int. J. Eng. Sci. 9, 587 (1971).

${ }^{2}$ H. F. Tiersten, J. Appl. Phys. 74, 3389 (1993).

${ }^{3} \mathrm{H}$. F. Tiersten, in Mechanics of Electromagnetic Materials and Structures, edited by J. S. Lee, G. A. Maugin, and Y. Shindo (ASME, New York, 1993), AMD-Vol. 161, MD-Vol. 42, pp. 21-34.

${ }^{4}$ Y. S. Zhou and H. F. Tiersten (unpublished).

${ }^{5}$ S. S. Rao and M. Sunar, Appl. Mech. Rev. 47, 113 (1994).

${ }^{6} \mathrm{C}$. A. Rogers and D. K. Barker, 31st AIAA-ASME-ASCE-AHS-ASC Structures, Structural Dynamics and Materials Conference, Long Beach, CA, April 2-4, 1990, pp. 2234-2241.

${ }^{7}$ II. S. Tzou and C. I. Tseng, J. Sound Vib. 137, 1 (1990).

${ }^{8}$ M. Sunar and S. S. Rao, in 33rd AIAA-ASME-ASCE-AHS-ASC Structures, Structural Dynamics and Materials Conference, Dallas, TX, April 13-15, 1992, pp. 890-895.

${ }^{9}$ T. R. Tauchert, J. Thermal Stresses 15, 25 (1992).

${ }^{10} \mathrm{Y}$. Y. Tang and $\mathrm{K}$. Xu (to be published).

${ }^{11}$ R. D. Mindlin, in Problems of Continuum Mechanics, N. I. Muskhelishvili 70th Birthday Volume (Society for Industrial and Applied Mathematics, Philadelphia, PA, 1961), pp. 282-291.

${ }^{12}$ J. F. Nye, Physical Properties of Crystals (Oxford University Press, London, 1957), p. 295.

${ }^{13}$ R. D. Mindlin, Int. J. Solids Struct. 10, 625 (1974).

${ }^{14}$ D. F. Nelson, Electric, Optic, and Acoustic Interactions in Dielectrics (Wiley, New York, 1979), pp. 490-513. 